\title{
Equation of State of the Two-Dimensional Hubbard Model
}

\author{
Eugenio Cocchi, ${ }^{1,2}$ Luke A. Miller, ${ }^{1,2}$ Jan H. Drewes, ${ }^{1}$ Marco Koschorreck, ${ }^{1}$ Daniel Pertot, ${ }^{1}$ \\ Ferdinand Brennecke, ${ }^{1}$ and Michael Köhl ${ }^{1, *}$ \\ ${ }^{1}$ Physikalisches Institut, University of Bonn, Wegelerstrasse 8, 53115 Bonn, Germany \\ ${ }^{2}$ Cavendish Laboratory, University of Cambridge, JJ Thomson Avenue, Cambridge CB3 OHE, United Kingdom
}

(Received 2 February 2016; published 25 April 2016)

\begin{abstract}
The subtle interplay between kinetic energy, interactions, and dimensionality challenges our comprehension of strongly correlated physics observed, for example, in the solid state. In this quest, the Hubbard model has emerged as a conceptually simple, yet rich model describing such physics. Here we present an experimental determination of the equation of state of the repulsive two-dimensional Hubbard model over a broad range of interactions $0 \lesssim U / t \lesssim 20$ and temperatures, down to $k_{B} T / t=0.63(2)$ using highresolution imaging of ultracold fermionic atoms in optical lattices. We show density profiles, compressibilities, and double occupancies over the whole doping range, and, hence, our results constitute benchmarks for state-of-the-art theoretical approaches.
\end{abstract}

DOI: 10.1103/PhysRevLett.116.175301

Ultracold fermionic atoms have emerged as a versatile platform to study strongly correlated spin- $1 / 2$ fermions since they submit to a precise microscopic description and superbly sensitive detection. This approach has shed new light, for example, on the crossover between a BoseEinstein condensate (BEC) of dimers and a BardeenCooper-Schrieffer (BCS)-type superconductor as well as on the universal physics of the unitary Fermi gas [1]. Among the remaining open questions are the properties of strongly interacting fermions in lattices, which have begun to be explored [2-10]. However, these investigations have not yet achieved the same level of accuracy in determining quantum phases and thermodynamic properties as those without lattice $[11,12]$. The experimental determination of the equation of state of the Hubbard model is of particular importance because, even with the most advanced theoretical methods, strongly correlated lattice models are notoriously hard to tackle $[13,14]$. Recently developed theoretical approximations of the two-dimensional Hubbard model $[15,16]$ provide predictions for a range of parameters; however, the inherent difficulty of simulating strongly correlated fermions has yet precluded the determination of a general phase diagram, and the predictions resulting from the approximations still require experimental verification.

The Hubbard model describes the two elementary processes of tunneling between neighboring lattice sites with amplitude $t$ and on-site interaction between two fermions of opposite spin with strength $U$. In a singleband approximation the Hubbard Hamiltonian reads

$H=-t \sum_{\langle i, j\rangle, \sigma}\left(\hat{c}_{i, \sigma}^{\dagger} \hat{c}_{j, \sigma}+\hat{c}_{j, \sigma}^{\dagger} \hat{c}_{i, \sigma}\right)+U \sum_{i} \hat{n}_{i, \downarrow} \hat{n}_{i, \uparrow}-\mu \sum_{i, \sigma} \hat{n}_{i, \sigma}$.
Here, $\hat{c}_{i, \sigma}\left(\hat{c}_{i, \sigma}^{\dagger}\right)$ denotes the annihilation (creation) operator of a fermion on lattice site $i$ in spin state $\sigma=\{\uparrow, \downarrow\}$, the bracket $\langle$,$\rangle denotes the restricted sum over nearest neigh-$ bors, $\hat{n}_{i, \sigma}=\hat{c}_{i, \sigma}^{\dagger} \hat{c}_{i, \sigma}$ is the number operator and $\mu$ is the chemical potential. One of the key signatures of the repulsive $(U>0)$ Hubbard model is the appearance of a Mott insulating state at half filling, i.e., for $n \equiv\left(\left\langle\hat{n}_{\uparrow}\right\rangle+\right.$ $\left.\left\langle\hat{n}_{\downarrow}\right\rangle\right) / 2=0.5$. The Mott insulator forms for $U \gg t, k_{B} T$, where $T$ is the temperature and $k_{B}$ is Boltzmann's constant. It is characterized by an occupation of one particle per lattice site and a gap against density excitations of order $U$.

Ultracold fermionic atoms in an optical lattice realize the Hubbard model $[2,8]$. In such experiments, the Hamiltonian parameters $t, U, \mu$, the temperature $T$, and the dimensionality are experimentally tunable, thus providing access to a large parameter range. Previously, investigations of the Hubbard model with ultracold atoms have mostly focused on the Mott insulator in three dimensions by detecting the global disappearance of doubly occupied sites $[3,5,17]$, the response to an external compression [4], the analysis of reconstructed density profiles [7], and global detection of local spin correlations [6,18]. Unlike homogeneous solid state systems, ultracold atoms are confined by an external trapping potential $V(\boldsymbol{r})$ leading to a spatially varying density distribution $n(\boldsymbol{r})$. Therefore, different quantum phases can coexist in different regions of the trap and their unique identification using global observables is often impossible. Conversely, with sufficient local resolution, the coexistence of different phases can, in principle, be used to sample a range of the phase diagram in a single experimental realization. For bosonic [19-21] and, recently, fermionic $[7,9,10]$ atoms in optical lattices the coexistence of different phases has been observed.

In this work we demonstrate high-resolution in situ imaging of a spin-balanced mixture of interacting spin- $1 / 2$ 
fermionic atoms in a single, two-dimensional layer of an optical lattice [see Fig. 1(a)]. By combining radiofrequency spectroscopy and absorption imaging we detect the in situ density distributions of singly occupied lattice sites ("singles"), $\left\langle\hat{n}_{\uparrow}-\hat{n}_{\uparrow} \hat{n}_{\downarrow}\right\rangle$, and of doubly occupied lattice sites ("doubles"), $\left\langle\hat{n}_{\uparrow} \hat{n}_{\downarrow}\right\rangle$ (for an extended description see the Supplemental Material [22]). This allows us to identify the two-dimensional Mott insulator and the metallic state spatially resolved. Crucially, our technique gives direct access to the equation of state [31] $n(\mu)$ and does not rely on density reconstruction, which can introduce numerical noise at small radii [7]. Our experiments cover the regimes from weak $(U / t \simeq 0)$ to strong $(U / t \simeq 20)$ interactions and, where available, we compare to state-of-the-art theories.
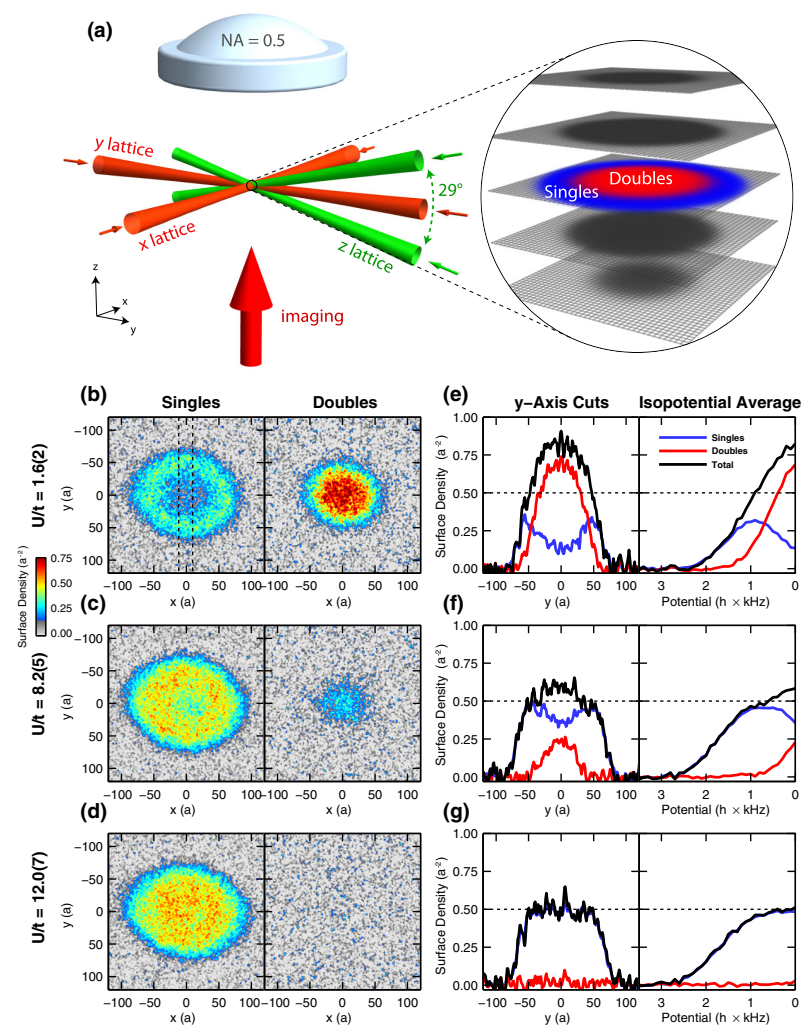

FIG. 1. Setup and in situ density profiles. (a) Experimental setup showing the arrangement of laser beams to create a stack of two-dimensional Hubbard models with ultracold atoms. Singles and doubles within a single layer are detected by combining radio-frequency spectroscopy and absorption imaging. (b)-(d) In situ density profiles of singles and doubles in the twodimensional Hubbard model for different interaction strengths. The images are averaged over $\sim 35$ repetitions of the experiment. The density profiles cross over from a metallic phase at weak interactions, $U / t=1.6$, to a flat-top Mott insulator without doubles at strong interaction $U / t=12.0$. (e)-(g) Singles, doubles, and total density data averaged over $-10 a \leq x \leq 10 a$ [see dashed lines in (b)] as well as evaluated along isopotential contours of the trapping potential.
In Fig. 1, we show examples of in situ density profiles for different interaction strengths [Figs. 1(b)-1(d)], together with cuts through the density distribution [Figs. 1(e)-1(g)]. For weak interactions, $U / t=1.6(2)$, the system is metallic with an inhomogeneous density distribution. In the center of the trap the density is highest and we find an accumulation of doubles. This dense core is surrounded by a low-density ring of singles [see Fig. 1(b)]. For intermediate interactions, $U / t=8.2(5)$, the doubles are suppressed by the increased interaction energy and, correspondingly, the size of the cloud increases [see Fig. 1(c)]. Finally, for strong interactions, $U / t=12.0(7)$, we do not observe any doubles and a pronounced plateau at filling $n=0.5$ forms, which signals the appearance of the Mott insulator [see Fig. 1(d)]. Employing the precise knowledge of the trapping potential $V(x, y)$ caused by the envelope of the optical lattice beams (going beyond the harmonic approximation used in Refs. [7,12,20,31], see Supplemental Material [22]) enables us to average the measured density along isopotential contours; see Figs. 1(e)-1(g).

We analyze our data in the framework of the local density approximation, which states that the local chemical potential $\mu(x, y)$ results from the chemical potential at the center $(x=y=0)$ of the cloud and the trapping potential by $\mu(x, y)=\mu(0,0)-V(x, y)$, and that the properties of the homogeneous system can be locally applied. The calibration of the central chemical potential $\mu(0,0)$ (based on the precise knowledge of $U$, see Supplemental Material [22]) is provided by the particle-hole symmetry of the Hubbard model, according to which the maximum of singles occurs at half filling, $n=0.5$, where the chemical potential is $\mu(n=0.5)=U / 2$. Combined with the knowledge of the potential, this allows us to convert the recorded density profiles into an equation of state $n(\mu)$ and to directly determine local thermodynamic properties of the gas $[12,20,31,32]$.

In Figure. 2(a), we show our experimental data of the equation of state $n(\mu)$ for different interaction strengths. The data show the crossover from a metal for weak interactions with a strong density variation across the region of half filling, to a Mott insulator with a plateau at half-filling for strong interactions. While the atom number remains approximately constant at $N \simeq 8.8(6) \times$ $10^{3}$ across different interaction strengths, there is a marked increase of the extent of the Mott-insulating region for larger $U / t$. We also note that the filling $n=0.5$ at a chemical potential of $\mu=U / 2$ is a fixed point of the equation of state, as theoretically expected. We fit the measured equation of state with data from numerical linked cluster expansion (NLCE) calculations [15], from which we determine the temperature of the gas. Our lowest temperature, $k_{B} T / t=0.63(2)$ at $U / t=8.2$, is already at the limit of validity of the numerical approximation, which is evident from the weak artificial oscillations of the 

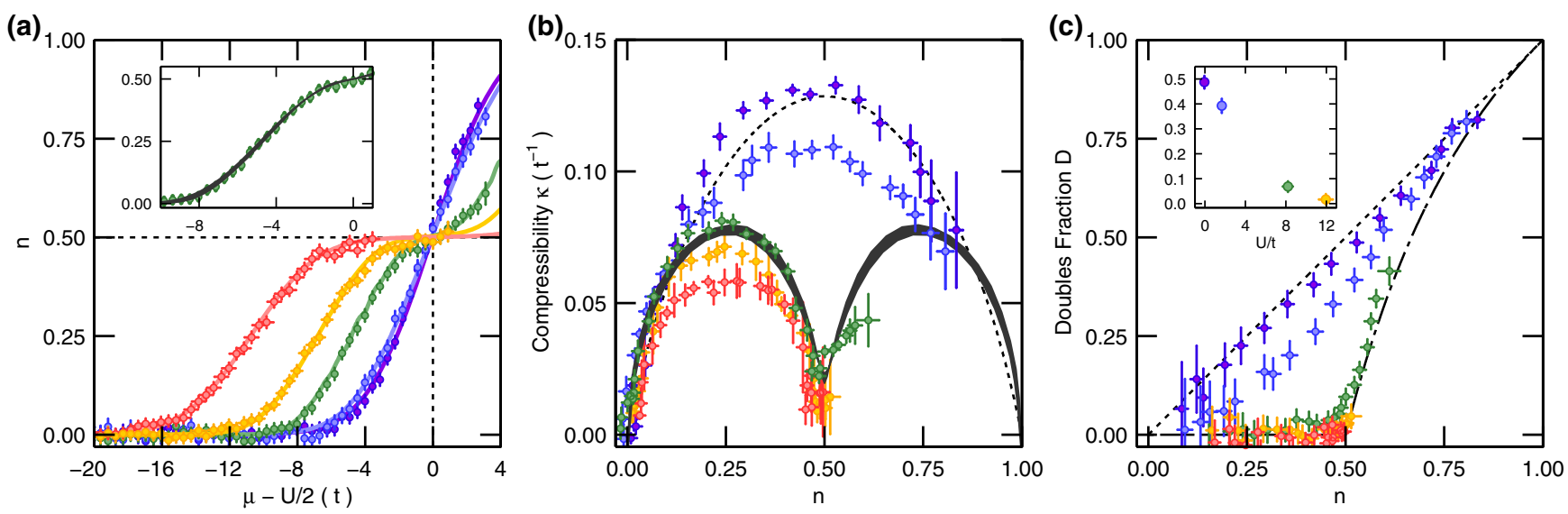

FIG. 2. Equation of state of the two-dimensional repulsive Hubbard model. Purple, $U / t=-0.2(3)$ for $k_{B} T / t=1.35(4)$; blue, $U / t=1.6(2)$ for $k_{B} T / t=1.19(4)$; green, $U / t=8.2(5)$ for $k_{B} T / t=0.63(2)$; yellow, $U / t=12.0(7)$ for $k_{B} T / t=0.92(6)$; red, $U / t=$ 19.5(1.3) for $k_{B} T / t=1.41(5)$. (a) Equation of state $n(\mu)$ vs interaction strength. Solid lines show fits using NLCE data [15] (purple, noninteracting Hubbard model) from which the temperature has been extracted. The inset shows the comparison with DCA data [16] for the temperature interval $0.55 \leq k_{B} T / t \leq 0.82$ at $U / t=8$. (b) Compressibility $\kappa$ vs filling. The dashed line shows the prediction of the noninteracting Hubbard model at $k_{B} T / t=1.4$ and the gray band the prediction from DCA as in (a). (c) Doubles fraction vs filling. The theoretical predictions for the noninteracting (dashed line) and infinitely repulsive (dashed-dotted line) Hubbard model are shown. The inset shows the behavior at $n=0.5$ vs interaction strength. The error bars show the standard errors.

theoretical $n(\mu)$ data near $n=0.25$ and above $n=0.5$. We also compare our data with the dynamical cluster approximation (DCA) [16] for $U / t=8$ [see inset of Fig. 2(a)], which confirms that they fall into the temperature interval $0.55 \leq k_{B} T / t \leq 0.82$. However, the available DCA data are too coarsely spaced in order to fit the temperature more accurately.

From the equation of state we compute the compressibility $\kappa=(\partial n / \partial \mu)$ shown in Fig. 2(b). The noninteracting gas exhibits a maximum compressibility of $\kappa=$ $0.133(3) t^{-1}$ at half filling $n=0.5$, which agrees with the numerical simulation of the homogeneous noninteracting Hubbard model at temperature $k_{B} T / t=1.4$ (dashed line). The approach from the metal to the Mott insulator is a crossover and therefore we expect a smooth change of the thermodynamic properties. For intermediate interactions, $U / t=8.2$, we already observe a significant reduction of the compressibility at $n=0.5$ and a return to a more compressible phase at fillings $n>0.5$. We compare this compressibility with data from DCA calculations [16] and find very good agreement. For strong interactions, $U / t \geq 12.0$, the compressibility is close to zero at half filling. For a system without disorder, a vanishing compressibility implies a gap against density (charge) excitations, and hence, combined with the observation of a plateau at half-filling, unequivocally demonstrates the observation of the Mott insulator in two dimensions.

Furthermore, in Fig. 2(c) we show the measured doubles fraction $D=2\left\langle\hat{n}_{\downarrow} \hat{n}_{\uparrow}\right\rangle /\left(\left\langle\hat{n}_{\uparrow}\right\rangle+\left\langle\hat{n}_{\downarrow}\right\rangle\right)=\left\langle\hat{n}_{\downarrow} \hat{n}_{\uparrow}\right\rangle / n$ vs filling and interaction strength. For the noninteracting gas, the spin-up and spin-down fillings are uncorrelated; hence, the doubles fraction simplifies to $D=n$ [dashed line in
Fig. 2(c)]. The data for the noninteracting gas agree with this prediction. In the limit of infinitely strong repulsive interactions, the number of doubles is completely suppressed if there are more lattice sites than particles, i.e., $D=0$ for $n \leq 0.5$, while for $n>0.5$ the number of doubles equals the excess of atoms above half filling, i.e., $D=2-1 / n$ [dashed-dotted curve in Fig. 2(c)]. We observe that even for $U / t=8.2$ the data are close to the infinite-interaction limit. For interaction strengths above this, the external compression provided by the trap is too weak to observe filling $n>0.5$. The inset shows the measured doubles fraction at half filling as a function of interaction strength $U / t$.

Finally, we investigate the equation of state for varying temperature at $U / t=8.2$. Around this interaction strength antiferromagnetic ordering is expected to occur at the highest transition temperature [33] of $k_{B} T / t \sim 0.3$ and thus knowledge of the thermodynamics will guide the approach to this state. In order to experimentally adjust the temperature, we heat the gas using a weak periodic modulation of the intensity of the horizontal lattice beams with a frequency close to twice the horizontal trapping frequency, followed by an equilibration time (see Supplemental Material [22]). In Fig. 3(a), we display how $n(\mu)$ varies with temperature. The distribution $n(\mu)$ gets broader and the compressibility at half-filling increases. The latter is detailed in Fig. 3(b) which shows how the Mott insulator melts and its compressibility increases from $\kappa=0.01 t^{-1}$ at low temperature to $\kappa=$ $0.03 t^{-1}$ at high temperature, in agreement with NLCE calculations (solid line). Moreover, in Fig. 3(c) we show the doubles fraction $D$ vs filling $n$ for varying temperatures 

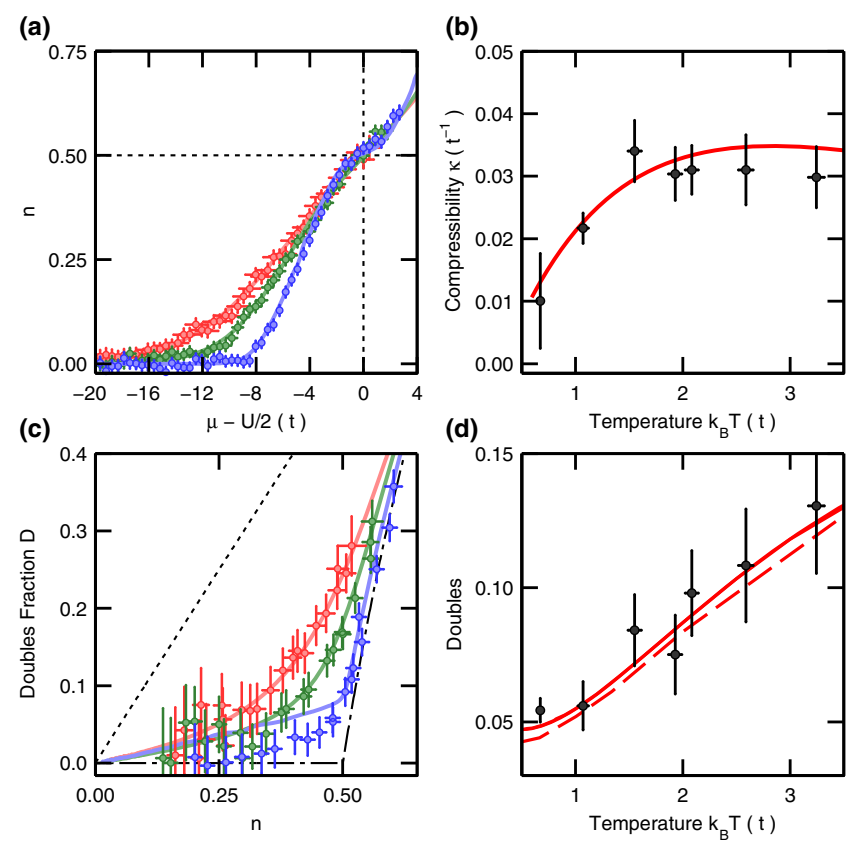

FIG. 3. Equation of state vs temperature at $U / t=8.2$. Blue, $\quad k_{B} T / t=0.67(3) ; \quad$ green, $\quad k_{B} T / t=1.55(6) ; \quad$ red, $k_{B} T / t=3.25(7)$. (a) Equation of state $n(\mu)$ for different temperatures with fits using NLCE data. (b) Compressibility at half filling vs temperature together with NLCE data. (c) Doubles fraction $D$ vs filling for different temperatures. (d) Doubles $\left\langle\hat{n}_{\uparrow} \hat{n}_{\downarrow}\right\rangle$ at half filling vs temperature. The solid line shows the coinciding predictions of NLCE [15] and DCA [16], and the dashed line the QMC prediction [33]. The error bars show the standard errors.

and find a considerable increase of doubles with temperature across all fillings. Some deviations between our experimental and the theoretical NLCE data are observed at low filling for low temperatures even though the total filling $n$ is in good agreement. In Fig. 3(d) we plot the doubles at half filling vs temperature and find that it increases from $0.054(5)$ at the lowest temperatures $k_{B} T / t=0.67(3)$ to $0.13(3)$ at $k_{B} T / t=3.25(7)$, in agreement with the results from NLCE [15], DCA [16], and quantum Monte Carlo (QMC) simulations [33]. Both, the increase of compressibility and the increase in doubles, signal the creation of thermally activated density excitations out of the lower Hubbard band. For the lowest temperatures our data are, within error, in agreement with the zero-temperature extrapolation of numerical theory calculations. This reflects that our measurements have reached the temperature limit for which density-dependent quantities are suited for studies of the thermodynamics of the Hubbard model. Extending our method to imaging both spin components individually could lead towards detection of magnetic order in the Hubbard model.

We thank J. L. Bohn, C. F. Chan, E. Gull, E. Khatami, C. Kollath, J. Leblanc, and M. Rigol for discussion. The work has been supported by BCGS, DFG, the Alexandervon-Humboldt Stiftung, EPSRC and ERC.

*michael.koehl@uni-bonn.de

[1] W. Zwerger, The BCS-BEC Crossover and the Unitary Fermi Gas (Springer, New York, 2012).

[2] M. Köhl, H. Moritz, T. Stöferle, K. Günter, and T. Esslinger, Phys. Rev. Lett. 94, 080403 (2005).

[3] R. Jördens, N. Strohmaier, K. Günter, H. Moritz, and T. Esslinger, Nature (London) 455, 204 (2008).

[4] U. Schneider, L. Hackermller, S. Will, T. Best, I. Bloch, T. A. Costi, R. W. Helmes, D. Rasch, and A. Rosch, Science 322, 1520 (2008).

[5] S. Taie, R. Yamazaki, S. Sugawa, and Y. Takahashi, Nat. Phys. 8, 825 (2012).

[6] R. A. Hart, P. M. Duarte, T.-L. Yang, X. Liu, T. Paiva, E. Khatami, N. T. Richard, T. Scalettar, D. A. Huse, and R. G. Hulet, Nature (London) 519, 211 (2015).

[7] P. M. Duarte, R. A. Hart, T.-L. Yang, X. Liu, T. Paiva, E. Khatami, R. T. Scalettar, N. Trivedi, and R. G. Hulet, Phys. Rev. Lett. 114, 070403 (2015).

[8] T. Esslinger, Annu. Rev. Condens. Matter Phys. 1, 129 (2010).

[9] D. Greif, M. F. Parsons, A. Mazurenko, C. S. Chiu, S. Blatt, F. Huber, G. Ji, and M. Greiner, Science 351, 953 (2016).

[10] C. Hofrichter, L. Riegger, F. Scazza, M. Höfer, D. R. Fernandes, I. Bloch, and S. Fölling, arXiv:1511.07287.

[11] M. J. H. Ku, A. T. Sommer, L.W. Cheuk, and M.W. Zwierlein, Science 335, 563 (2012).

[12] N. Navon, S. Nascimbene, F. Chevy, and C. Salomon, Science 328, 729 (2010).

[13] A. Georges, G. Kotliar, W. Krauth, and M. J. Rozenberg, Rev. Mod. Phys. 68, 13 (1996).

[14] P. A. Lee, N. Nagaosa, and X.-G. Wen, Rev. Mod. Phys. 78, 17 (2006).

[15] E. Khatami and M. Rigol, Phys. Rev. A 84, 053611 (2011).

[16] J. P. F. LeBlanc and E. Gull, Phys. Rev. B 88, 155108 (2013).

[17] R. Jördens, L. Tarruell, D. Greif, T. Uehlinger, N. Strohmaier, H. Moritz, T. Esslinger, L. De Leo, C. Kollath, A. Georges, V. Scarola, L. Pollet, E. Burovski, E. Kozik, and M. Troyer, Phys. Rev. Lett. 104, 180401 (2010).

[18] D. Greif, T. Uehlinger, G. Jotzu, L. Tarruell, and T. Esslinger, Science 340, 1307 (2013).

[19] W. S. Bakr, A. Peng, M. E. Tai, R. Ma, J. Simon, J. I. Gillen, S. Flling, L. Pollet, and M. Greiner, Science 329, 547 (2010).

[20] N. Gemelke, X. Zhang, C.-L. Hung, and C. Chin, Nature (London) 460, 995 (2009).

[21] J. F. Sherson, C. Weitenberg, M. Endres, M. Cheneau, I. Bloch, and S. Kuhr, Nature (London) 467, 68 (2010).

[22] See Supplemental Material http://link.aps.org/supplemental/ 10.1103/PhysRevLett.116.175301, which includes Refs. [23-30].

[23] M. Dolfi, A. Kantian, B. Bauer, and M. Troyer, Phys. Rev. A 91, 033407 (2015).

[24] M. Garwood and L. DelaBarre, J. Magn. Reson. 153, 155 (2001).

[25] T. Rom, T. Best, D. van Oosten, U. Schneider, S. Fölling, B. Paredes, and I. Bloch, Nature (London) 444, 733 (2006). 
[26] G. Reinaudi, T. Lahaye, Z. Wang, and D. Guéry-Odelin, Opt. Lett. 32, 3143 (2007).

[27] Z. Idziaszek and T. Calarco, Phys. Rev. A 74, 022712 (2006).

[28] P.-I. Schneider, S. Grishkevich, and A. Saenz, Phys. Rev. A 80, 013404 (2009).

[29] M. J. Mark, E. Haller, K. Lauber, J. G. Danzl, A. J. Daley, and H.-C. Nägerl, Phys. Rev. Lett. 107, 175301 (2011).
[30] L. De Leo, J.-S. Bernier, C. Kollath, A. Georges, and V. W. Scarola, Phys. Rev. A 83, 023606 (2011).

[31] T.-L. Ho and Q. Zhou, Nat. Phys. 6, 131 (2010).

[32] K. V. Houcke, F. Werner, E. Kozik, N. Prokofev, B. Svistunov, M. J. H. Ku, A. T. Sommer, L. W. Cheuk, A. Schirotzek, and M. W. Zwierlein, Nat. Phys. 8, 366 (2012).

[33] T. Paiva, R. Scalettar, M. Randeria, and N. Trivedi, Phys. Rev. Lett. 104, 066406 (2010). 